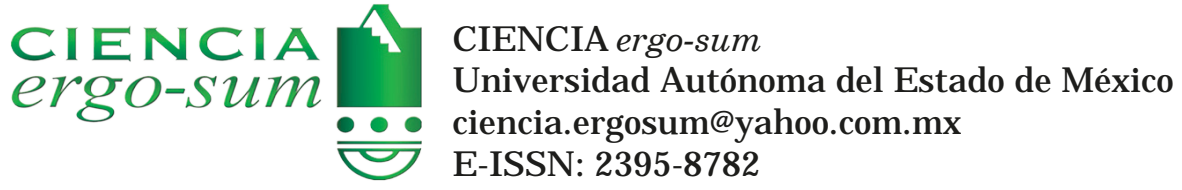

\title{
Desechos radiactivos de vida media corta en hospitales públicos en México: estado actual
}

Torres-Carranza, Mónica Alexia; Ortiz-Oliveros, Huemantzin Balan

Desechos radiactivos de vida media corta en hospitales públicos en México: estado actual

CIENCIA ergo-sum, vol. 26, núm. 2, julio-octubre 2019| e54

Universidad Autónoma del Estado de México, México

Esta obra está bajo una Licencia Creative Commons Atribución-NoComercial-SinDerivar 4.0 Internacional.

Torres-Carranza, M. A. y Ortiz-Oliveros, H. B. (2019). Desechos radiactivos de vida media corta en hospitales públicos en México: estado actual. CIE NCIA ergo-sum, 26(2). https://doi.org/10.30878/ces.v26n2a8 


\title{
Desechos radiactivos de vida media corta en hospitales públicos en México: estado actual
}

Short-lived radioactive wastes in Public Hospitals in Mexico: Current state

\author{
Mónica Alexia Torres-Carranza \\ Universidad Autónoma del Estado de México, México \\ monikalexia10@gmail.com
}

Recepción: 17 de mayo de 2018

Aprobación: 17 de julio de 2018

Huemantzin Balan Ortiz-Oliveros

Instituto Nacional de Investigaciones Nucleares, México

hbortizo@uaemex.mx

\section{RESUMEN}

Se presenta el estado de la gestión de los desechos radiactivos de vida media corta en hospitales públicos. Se revisó el marco regulatorio que rige la gestión de desechos, así como las instituciones médicas que producen la mayor cantidad y que también utilizan radioisótopos. Como instrumento de recopilación de información, se empleó el cuestionario, el cual fue formulado considerando criterios como capacitación, generación de desechos, entre otros. Los resultados evidenciaron que se generan $0.08 \mathrm{~kg}$ de desechos sólidos por unidosis al año. Los desechos líquidos no son cuantificados. Asimismo, se identificó que las instituciones cumplen parcialmente con la normatividad sin que implique un riesgo radiológico. La carencia de un plan de gestión es el causal de las áreas de oportunidad observadas.

Palabras Clave: hospitales, desechos radiactivos, radionúclidos, gestión, plan de gestión, vida media corta.

\begin{abstract}
This work presents the state of short half-life radioactive waste management in public hospitals. The national regulatory framework that determines radioactive waste management was identified. Likewise, it was identified and established the main medical institutions that use radioisotopes and produces the most amount of radioactive waste. The methodological collection instrument was the questionnaire. This was designed considering criteria such as: personnel training, waste generation, among others. The results showed that $0.08 \mathrm{~kg}$ of single-dose solid radioactive wastes are generated per year. Liquid radioactive waste is not quantified. The evidence obtained allowed us to identify that medical institutions partially comply with regulations without involving a radiological risk to personnel, patients or the environment. The absence of an institutional radioactive waste management plan is the cause of the areas of opportunity observed.
\end{abstract}

KEYWORDS: hospitals, radioactive waste, radionuclides, management, management plan, short half-life.

\section{INTRODUCCIÓN}

Actualmente los países en desarrollo enfrentan diferentes retos de índole social, económico, político e incluso de seguridad pública, todos ellos complejos y que además requieren del desarrollo de políticas públicas específicas y el compromiso de todos los sectores de la sociedad y gobierno. Por otro lado, existen problemas que si bien no tienen carácter de seguridad nacional, son tan sensibles como éstos. Como ejemplo, se tiene la necesidad de mejorar la atención de salud, estimular la producción agrícola, aumentar la seguridad alimentaria o de optimizar la gestión de los recursos naturales, todas posibles gracias al uso de los avances tecnológicos que el mundo contemporáneo ofrece. La tecnología nuclear, por ejemplo, aporta soluciones eficaces y sustentables, 
además de ofrecer una amplia gama de aplicaciones en áreas como la energética, médica, alimentaria, industrial o de investigación.

El desarrollo de la tecnología nuclear en México, aunque controvertida, data de la década de 1950 con la aplicación de material radiactivo (radioisótopos) y en 1954 con la creación del primer laboratorio especializado en medicina nuclear en el Hospital General de La Raza del Instituto Mexicano del Seguro Social (IMSS) (Pichardo, 2015). Los radioisótopos tienen diversas aplicaciones en el campo de la medicina nuclear y se extienden a todas las ramas de la medicina: oncología, endocrinología, hematología, cardiología, entre otras; de igual forma, se emplean técnicas como la radioterapia para el tratamiento de tumores malignos (Jawerth, 2014; Skromne, 2010).

Décadas más tarde, en 1970, el país inicia su proyecto transexenal más importante: la construcción y funcionamiento de la primera central nucleoeléctrica (Villareal, 2011). Sin embargo, la inestabilidad financiera de los años posteriores entorpece el proyecto y es hasta 1988 que estos sueños se ven cristalizados al poner en marcha la Unidad I de la Central Nucleoeléctrica Laguna Verde (CLV). En septiembre de 1994 se pone en funcionamiento la Unidad II de la CLV (Ortiz, 2002). Ambas unidades representan más de $2.8 \%$ de la capacidad eléctrica instalada en el país y contribuyen con 5\% de la producción eléctrica nacional (Díaz, 2015).

Por otro lado, el uso de materiales radiactivos se encuentra generalizado en el sector industrial, en donde se utiliza para controlar y regular los procesos, lo que permite mejorar la calidad del producto, aprovechar las materias primas y asegurar la fiabilidad de las líneas de producción (OIEA, 2015; Machi et al., 1983). Asimismo, la tecnología nuclear tiene injerencia en la investigación de procesos, mezclado, mantenimiento de equipos, el estudio del desgaste y corrosión de instalaciones y maquinaria, así como en la fabricación de plásticos y en la esterilización de productos diversos, entre otros.

A través del tiempo se ha estigmatizado el uso de la tecnología nuclear debido a sus aplicaciones con fines bélicos en eventos como el de Three Mile Island, Chernóbil y recientemente Fukushima. Sin embargo, es importante resaltar que la tecnología nuclear con fines pacíficos forma parte de la vida cotidiana, pues ofrece alternativas eficaces para las diversas áreas de aplicación. Tal es el caso de la medicina nuclear, la cual utiliza pequeñas cantidades de sustancias radiactivas con radioisótopos, por lo regular de vida media corta, ya sea en fuentes selladas o abiertas. Estas sustancias son utilizadas en radioinmunoensayos, radiofármacos, procedimientos de diagnóstico, radioterapia (teleterápia y braquiterapia) y en investigación (OIEA, 2001). En los procedimientos de diagnóstico se emplea la mayor cantidad de material radiactivo (radiofármacos), pues incluye la realización de pruebas funcionales, morfológicas, dinámicas, morfofuncionales y analíticas basadas en principios bioquímicos, fisiológicos y fisiopatológicos encaminadas a conseguir un mejor conocimiento y comprensión de la estructura y función del estado de salud del paciente (MSSSI, 1996). Estos radiofármacos se caracterizan por tener pequeñas cantidades del principio activo, conocido como trazador, que se marca con un radionúclido ocasionando que emitan una dosis de radiación (Cortés y Esteban, 2003). Cuando son utilizados con el propósito de diagnóstico, se aprovecha la propiedad emisora de los radioisótopos para detectarlos a distancia; cuando la intención es terapéutica, se aprovecha el efecto deletéreo que la radiación tiene sobre las células (Chain e Illanes, 2015).

Tal como se ha señalado, son innegables los aspectos positivos que el uso de la tecnología nuclear tiene en la sociedad a través de su aplicación en la medicina. Sin embargo, independientemente de los beneficios que aporta, tiene el inconveniente de generar desechos radiactivos que requieren de una gestión apropiada con el objeto de minimizar los riesgos a la salud del personal expuesto, pacientes, sociedad y el ambiente. De hecho, según fuentes gubernamentales se estima que en el país $90 \%$ de los desechos radiactivos provienen de aplicaciones energéticas, mientras que 10\% restante se produce en las no energéticas; de estos últimos, $89 \%$ proviene del sector médico (CNSNS, 2015).

Diversos autores han elaborado investigaciones para analizar el estatus de la gestión de los desechos radiactivos generados en aplicaciones médicas. Por ejemplo, Duran y Munar (2006) llevaron a cabo un diagnóstico de 
seguridad ambiental para la gestión de desechos radiactivos originados por la medicina nuclear en la ciudad de Bogotá con el fin de exponer el cumplimiento normativo de los centros con servicio de medicina nuclear. Entre sus conclusiones refieren que el sector de la medicina nuclear distrital de Bogotá posee una vaga comprensión de los fundamentos técnicos de las etapas de la gestión de desechos radiactivos. Álvarez y Moreno (2010) implementaron un plan de gestión integral de residuos hospitalarios y similares en el hospital Chapinero E.S.E. en Colombia. Por su parte, Álvarez Rico (2010) presentó una metodología para la caracterización de los desechos radiactivos provenientes del área médica nuclear en México y de este modo contar con los elementos para la toma de decisiones en la gestión posibilitando su dispensa inmediata o propiciando un cálculo adecuado del tiempo requerido en decaimiento.

Estos trabajos tienen en común que no consideran la cuantificación de los desechos radiactivos generados, así como las actividades administrativas y operacionales requeridas para su gestión. En lo que respecta a México no se encontró información referente al manejo de los desechos radiactivos del sector salud que permitiera dimensionar su situación. En este contexto, el artículo presenta el estado situacional de la generación de desechos radiactivos de vida media corta en hospitales públicos en México.

\section{Materiales y métodos}

Este artículo se llevó a cabo en tres fases. La primera de ellas consistió en establecer y revisar el marco regulatorio nacional que rige la gestión de los desechos radiactivos partiendo de la ley reglamentaria del Artículo 27 Constitucional en Materia Nuclear, el Reglamento General de Seguridad Radiológica (RGSR) y las normas oficiales mexicana en materia nuclear.

El siguiente paso consistió en definir las unidades de estudio (delimitación de la población), es decir, las instituciones médicas que poseen servicio de medicina nuclear. Esta información se obtuvo a través de la Secretaría de Salud. Para seleccionar la muestra de estudio, se tomó como criterio de elección a las instituciones médicas calificadas como las principales generadoras de desechos radiactivos hospitalarios. Esta información se solicitó a la Comisión Nacional de Seguridad Nuclear y Salvaguardas (CNSNS). Ambas solicitudes se formalizaron a través de la plataforma nacional de transparencia del Instituto Nacional de Transparencia, Acceso a la Información y Protección de Datos Personales (INAI).

Una vez identificadas las unidades de estudio, se definió el instrumento de recolección de datos. En particular, este trabajo optó por el cuestionario: instrumento de medición tanto cualitativo como cuantitativo utilizado ampliamente en investigaciones sociales, económicas, entre otras. El cuestionario permite traducir las dimensiones en elementos medibles, es decir, pasar de las dimensiones a los indicadores y de éstos a las preguntas (Escofet et al. 2016; Galtung, 1967). Los criterios utilizados para su elección fueron: a) la disponibilidad de recursos económicos, humanos y materiales, $b$ ) la dispersión geográfica de la población bajo estudio y c) la funcionalidad en su aplicación y confiabilidad de la información obtenida. El cuestionario fue formulado con preguntas abiertas y cerradas considerando los siguientes criterios (figura 1): a) capacitación del Personal Ocupacionalmente Expuesto (POE) involucrado en el manejo del material radiactivo, $b$ ) identificación de los radioisótopos utilizados en el área médica, $c$ ) cantidad e identificación de los desechos radiactivos generados por las instituciones médicas sujetas a estudio, $d$ ) características de los insumos utilizados para la segregación y recolección de los desechos radiactivos, así como e) la infraestructura para realizar el decaimiento o almacenamiento temporal de los desechos. El cuestionario quedó plasmado en un formato que permitió recopilar la información de las variables sujetas a estudio de manera sistemática y ordenada. Este formato, junto con las solicitudes de información, se envió a cada una de las unidades de enlace de las instituciones médicas seleccionadas a través de la plataforma nacional de transparencia del INAI. 


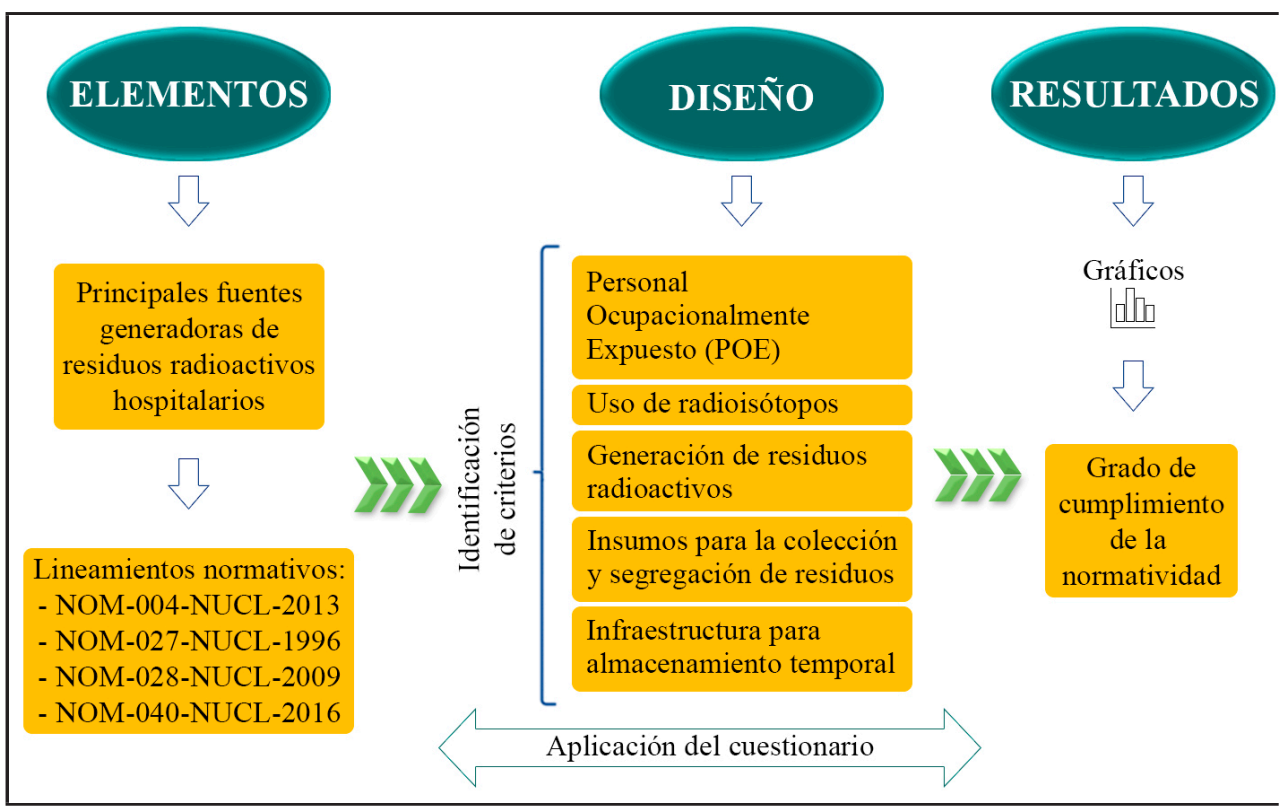

FIGURA 1

Proceso de elaboración del instrumento de investigación

Fuente: elaboración propia.

\section{Resultados y discusiones}

Producto de la solicitud de información realizada a la CNSNS a través del INAI, fue posible determinar que en el país se tiene el registro de 441 licencias de operación de fuentes radiactivas con fines médicos: 229 amparan el uso de fuentes abiertas y 212 la operación de fuentes selladas. Por otra parte, la Secretaría de Salud señaló que existen 52 instituciones médicas de carácter público con servicios de medicina nuclear que utilizan fuentes de radiación. Según la CNSNS, únicamente 22 de las 52 instituciones son consideradas como las principales generadoras de desechos radiactivos hospitalarios (tabla 1 ).

TABLA 1

Principales instituciones generadoras de desechos radiactivos

\begin{tabular}{|llc}
\hline Clave & \multicolumn{1}{c}{ Instalación médica } & $\begin{array}{c}\text { Años de operación y servicio } \\
\text { en medicina nuclear }\end{array}$ \\
\hline H1 & Centro Médico Nacional 20 de noviembre & 55 \\
H2 & Hospital Juárez de México & 22 \\
H3 & Hospital Infantil de México Federico Gómez & 20 \\
H4 & Unidad Médica de Alta Especialidad, Hospital de Especialidades Centro & ND \\
H5 & Médico Nacional de Occidente. & 44 \\
H6 & Instituto Nacional de Pediatría & 42 \\
H7 & Instituto Nacional de Cancerología & 36 \\
H8 & UMAE, Hospital de Especialidades Dr. Antonio Fraga Mouret CMN La & 45 \\
H9 & Raza & Hospital General de México Dr. Eduardo Liceaga \\
H10 & Instituto Nacional de Ciencias Médicas y Nutrición Salvador Zubirán & ND \\
H11 & UMAE, Hospital de Oncología CMN Siglo XXI & 63 \\
H12 & UMAE, Hospital de Especialidades CMN Siglo XXI Bernardo Sepúlveda & 23 \\
\hline
\end{tabular}


TABLA 1 (continúa)

\begin{tabular}{|c|c|c|}
\hline H13 & $\begin{array}{l}\text { UMAE, Hospital de Especialidades CMN Gral. de Div. Manuel Ávila } \\
\text { Camacho }\end{array}$ & 40 \\
\hline H14 & Instituto Nacional de Cardiología Ignacio Chávez & 40 \\
\hline H15 & Hospital Regional Lic. Adolfo López Mateos & 38 \\
\hline H16 & UMAE, Hospital de Cardiología, Centro Médico Nacional Siglo XXI & 38 \\
\hline H17 & $\begin{array}{l}\text { Unidad Médica de Alta Especialidad, Hospital de Especialidades No. 25, } \\
\text { Centro Médico Nacional del Noreste }\end{array}$ & 36 \\
\hline H18 & $\begin{array}{l}\text { UMAE, Hospital de Especialidades del Centro Médico Nacional, Ignacio } \\
\text { García Téllez }\end{array}$ & 35 \\
\hline H19 & Hospital de Especialidades No. 71, Centro Médico Nacional, Torreón & 7 \\
\hline $\mathrm{H} 20$ & Instituto Nacional de Enfermedades Respiratorias Ismael Cosío Villegas & 39 \\
\hline $\mathrm{H} 21$ & $\begin{array}{l}\text { Unidad Médica de Alta Especialidad (UMAE), Hospital de Especialida- } \\
\text { des No. } 1 \text { Centro Médico Nacional Bajío }\end{array}$ & 29 \\
\hline $\mathrm{H} 22$ & $\begin{array}{l}\text { UMAE, Hospital de Cardiología No. 34, Centro Médico Nacional del } \\
\text { Noreste }\end{array}$ & 20 \\
\hline
\end{tabular}

Fuente: elaboración propia con base en información proporcionada por la CNSNS, 2016.

Una vez identificadas las unidades de estudio, se envió el cuestionario a las 22 instituciones médicas. La información recibida fue sistematizada y ordenada para su posterior análisis. Al respecto, en la tabla 1 se incluyen los años de operación y servicio en medicina nuclear de las 22 instituciones estudiadas. En la tabla se puede observar que las instituciones con mayor antigüedad en el manejo de unidades de medicina nuclear son el Instituto Nacional de Ciencias Médicas y Nutrición Salvador Zubirán con 63 años, seguido del Centro Médico Nacional 20 de noviembre con 55 años, Hospital de Especialidades CMN Siglo XXI Bernardo Sepúlveda Gutiérrez con 50 años, el Hospital de Especialidades Dr. Antonio Fraga Mouret CMN La Raza con 45 años y el Instituto Nacional de Pediatría con 44 años. El resto se encuentra en un intervalo entre 20 y 40 años. Dos instituciones no proporcionaron información. Los datos obtenidos ofrecen un panorama general de los años de experiencia que cada institución tiene en el uso de material radiactivo, la cual se esperaría estuviera correlacionada con la eficiencia en la gestión de sus desechos radiactivos.

\section{Capacitación del Personal ocupacionalmente expuesto}

Respecto a las cualificaciones que tiene el personal que labora en el área de medicina nuclear, destacan las de médicos nucleares, técnicos en medicina nuclear, químicos, enfermeras, físicos médicos, laboratoristas y, en algunos casos, personal de intendencia. El personal listado es el responsable de la recepción y manipulación del material radiactivo, así como de la gestión de los desechos radiactivos generados; por lo tanto, es el más vulnerable a las radiaciones ionizantes o a la incorporación de material radiactivo en caso de no realizar estas operaciones de manera adecuada. Cada institución debe demostrar que su personal, independientemente del nivel académico, tiene la capacitación y adiestramiento requerido por el RGSR. En los resultados obtenidos, las 22 instituciones señalan que el personal está incluido en la Licencia de Operación y que tienen las cualificaciones, al menos teóricas, para manipular materiales radiactivos. No obstante, dos hospitales señalaron que el personal de intendencia realiza la manipulación de los desechos radiactivos, pero no lo incluyen en las licencias y autorizaciones de operación. $\mathrm{Al}$ respecto, enfatizan que este personal es capacitado internamente en materia de protección radiológica. Cabe resaltar que en términos legales esto representa un incumplimiento a las licencias de operación y al RGSR, pero sobre todo incrementa de manera innecesaria los riesgos de exposición del personal de intendencia. 


\section{USO DE RADIOFÁRMACOS Y GENERACIÓN DE DESECHOS RADIACTIVOS}

Referente al uso de radiofármacos y generación de desechos radiactivos, de acuerdo con el análisis de la información proporcionada por la CNSNS, en México existen 29 radioisótopos autorizados para procedimientos de medicina nuclear: ${ }^{13} \mathrm{~N},{ }^{15} \mathrm{O},{ }^{18} \mathrm{~F},{ }^{22} \mathrm{Na},{ }^{32} \mathrm{P},{ }^{35} \mathrm{~S},{ }^{51} \mathrm{Cr},{ }^{55} \mathrm{Fe},{ }^{57} \mathrm{Co},{ }^{60} \mathrm{Co},{ }^{67} \mathrm{Ga},{ }^{68} \mathrm{Ga},{ }^{99} \mathrm{Sr},{ }^{90} \mathrm{Sr},{ }^{90} \mathrm{Y},{ }^{99} \mathrm{~m} \mathrm{Tc}$, ${ }^{99} \mathrm{Mo},{ }^{111} \mathrm{In},{ }^{125} \mathrm{I},{ }^{131} \mathrm{I},{ }^{133} \mathrm{Ba},{ }^{137} \mathrm{Cs},{ }^{153} \mathrm{Sm},{ }^{177} \mathrm{Lu},{ }^{188} \mathrm{Re},{ }^{192} \mathrm{Ir},{ }^{198} \mathrm{Au},{ }^{201} \mathrm{Tl}$ y ${ }^{223} \mathrm{Ra}$.

En la gráfica 1 se observa que los radionúclidos más utilizados en aplicaciones médicas y en las 22 instituciones

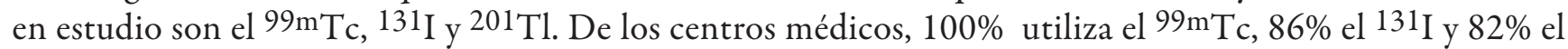
${ }^{201} \mathrm{Tl}$. El $99 \mathrm{~m}$ Tc es un radionúclido emisor gamma de vida media corta $(6 \mathrm{~h})$, con una gamma significativa de $140.51 \mathrm{keV}$. Este radionúclido es considerado como el más importante en el campo de la medicina, se emplea en la detección de padecimientos, en la formación de imágenes del esqueleto y el miocardio, así como para otros numerosos estudios médicos especializados (Henriques, 2014). El 131 I es un emisor beta con una vida media de ocho días que se utiliza para monitorear la actividad de la glándula tiroides, el cáncer de tiroides y para la formación de imágenes de la tiroides (Henriques, 2014). Tiene la peculiaridad de dispersarse con facilidad cuando se maneja inadecuadamente. El ${ }^{201} \mathrm{Tl}$ tiene una vida media de tres días y se usa con fines de imagen cardiovascular. La identificación del núclido y el conocimiento de sus características fisicoquímicas y radiológicas son aspectos importantes en la gestión de los desechos, ya que permiten establecer el tiempo de almacenamiento temporal para el decaimiento radiactivo. Es importante resaltar que en las instituciones médicas se utilizan fuentes selladas con radioisótopos con vida media superior a un año (gráfica 1) tales como el ${ }^{137} \mathrm{Cs}$ y ${ }^{60} \mathrm{Co}$. El ${ }^{137} \mathrm{Cs}$, vida media de 30.08 años, suele utilizarse para la irradiación de plasma sanguíneo, unidades de braquiterapia y en menor proporción en unidades de teleterápia contra el cáncer. El ${ }^{60}$ Co, vida media 5.3 años, se emplea principalmente en teleterápia. Este tipo de fuentes después de cumplir con su vida útil tiene que ser gestionada a través del Instituto Nacional de Investigaciones Nucleares (ININ) para su acondicionamiento y almacenamiento temporal. Asimismo, las fuentes selladas gastadas de ${ }^{192} \operatorname{Ir}$ (73.8 días) tienen que ser gestionadas para su acondicionamiento y almacenamiento temporal en el ININ. Esta institución es la encargada de la gestión de los desechos radiactivos y fuentes selladas gastadas que contienen radionúclidos de vida superior a un año.

Se solicitó a las instituciones médicas el promedio mensual de unidosis utilizadas en sus pacientes en 2015, así como la cantidad de desechos radiactivos generados en el mismo periodo. Hay que señalar que las instituciones médicas consultadas no realizan la cuantificación de los desechos radiactivos por radionúclido. En este sentido, los resultados se muestran en la gráfica 2, en donde se observa que $72.7 \%$ de las instituciones atendieron un promedio mensual de 140 a 646 pacientes, entre las cuales destacan el Instituto Nacional de Cancerología con 646 pacientes, el Hospital de Especialidades CMN Siglo XXI Bernardo Sepúlveda Gutiérrez con 580 pacientes, el Hospital de Especialidades Centro Médico Nacional de Occidente con 510 pacientes, el Centro Médico Nacional 20 de noviembre y el Hospital de Especialidades CMN Gral. de Div. Manuel Ávila Camacho con 400 pacientes al mes respectivamente, mientras que cuatro instituciones no proporcionaron información. Con relación a la cantidad de los desechos radiactivos sólidos que se generaron por mes a causa de la aplicación de unidosis, los resultados indican que las cantidades varían drásticamente de un hospital a otro. Al respecto, el Hospital de Cardiología Centro Médico Nacional Siglo XXI es el mayor generador con $240 \mathrm{~kg}$ al mes. De las instituciones médicas, $31.8 \%$ produce entre $10-50 \mathrm{~kg}$ al mes. En este aspecto destaca el a) Hospital de Especialidades No. 71, Centro Médico Nacional, Torreón, con $50 \mathrm{~kg}$, b) el Hospital de Especialidades Dr. Antonio Fraga Mouret CMN La Raza con $40 \mathrm{~kg}$, c) el Instituto Nacional de Ciencias Médicas y Nutrición Salvador Zubirán con $25 \mathrm{~kg}$, d) el Hospital de Especialidades Centro Médico Nacional de Occidente e Instituto Nacional de Cardiología Ignacio Chávez con 15 kg y e) el Hospital de Especialidades del Centro Médico Nacional, Ignacio García Téllez con 10 kg. 
En suma, las instituciones consultadas aplicaron aproximadamente 5300 unidosis al mes y generaron 450 $\mathrm{kg}$ de desechos en el mismo periodo, es decir, se produjeron $0.08 \mathrm{~kg}$ de desechos promedio por unidosis en 2015. Esta tasa de generación baja de desechos puede asociarse a dos factores: a) existe la práctica de que las instituciones entreguen al proveedor del radiofármaco los remanentes de las jeringas, las jeringas y contenedores del radioisótopo y $b$ ) a la inadecuada cuantificación y registro de los desechos producidos. En este contexto, y con información parcial obtenida de instituciones privadas ( 140 unidosis aplicadas al mes), se estima que a nivel nacional en 2015 se generaron $7080 \mathrm{~kg}$ de desechos radiactivos sólidos de vida media corta.

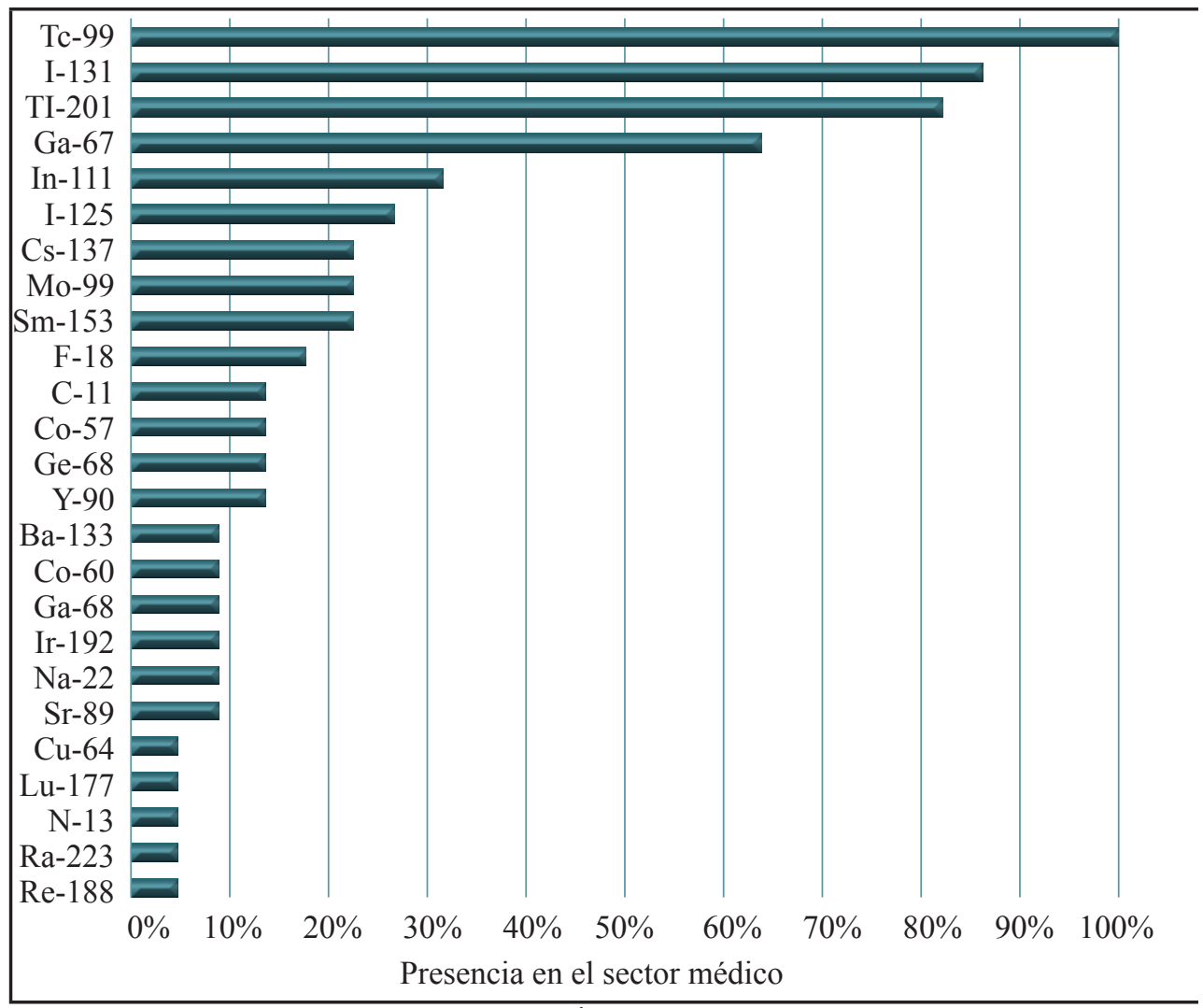

\section{GRÁFICA 1}

Radioisótopos de uso médico utilizados en las instituciones de salud en México Fuente: elaboración propia (2016).

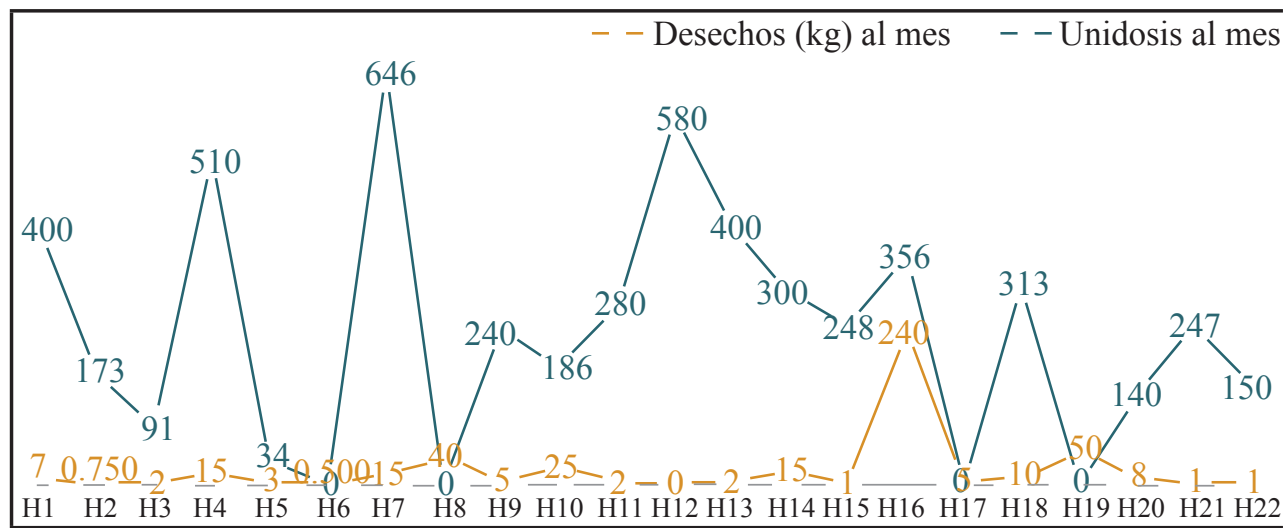

GRÁFICA 2

Unidosis aplicadas y cantidad de desechos generados

Fuente: elaboración propia (2016). 
En cuanto a su composición, los desechos radiactivos sólidos están conformados por insumos médicos que se contaminan durante la preparación y administración del radiofármaco; en ellos pueden identificarse agujas, jeringas, gasas, torundas, guantes, pañales, llaves de tres vías, extensión de venoclisis, papel absorbente y ropa hospitalaria. Todas las instituciones generan jeringas y agujas, $77 \%$ torundas y guantes, mientras que $55 \%$ gasas. En menor porcentaje se produce papel absorbente y ropa de hospital con 27 y $18 \%$ respectivamente.

Por otro lado, se pudo establecer que $64 \%$ de las instituciones médicas genera desechos líquidos radiactivos producto de las excretas de los pacientes tratados con terapia metabólica (131I), mientras que 36\% restante dice no producir este tipo de desecho. No se encontró evidencia de una adecuada cuantificación de estos desechos.

\section{Segregación Y RECOLECCión DE DESEChos RADIACTIVOS}

Con la finalidad de conocer cómo las instituciones médicas realizan la segregación y recolección de desechos radiactivos, se realizó el planteamiento de diferentes cuestionamientos para conocer los criterios utilizados, las características físicas de los contenedores empleados para la recolección y la información contenida en el etiquetado, entre otros.

Existen criterios que sirven de pilar al momento de establecer la clasificación de los desechos radiactivos tales como su estado físico (líquido, sólido y gaseoso), el tipo de radiación emitida (alfa, beta y gamma), el periodo de semidesintegración (corta, media y alta), la actividad específica y la radiotoxicidad. Los centros de salud pueden generar desechos radiactivos sólidos y líquidos, los sólidos suelen ser fuentes selladas gastadas, materiales compactables (papeles, guantes, etcétera) y materiales no compactables (madera, metales, agujas hipodérmicas, etcétera) (OIEA, 2001). Los líquidos pueden ser de tipo acuoso (soluciones salinas, básicas o ácidas) y orgánicos (solventes orgánicos y mezclas de líquidos de centelleo). Estos pueden ser emisores alfa, beta o gamma con vida media de minutos, horas o hasta 30 años como máximo. Los radionúclidos de vida media superior a 30 años no son comunes en aplicaciones médicas.

La información recabada mostró que las instituciones médicas segregan los desechos radiactivos tomando en cuenta tres aspectos: $a$ ) el estado físico, $b$ ) la vida media del radionúclido del material contaminado y $c$ ) la radiación emitida. Al respecto, $86 \%$ de las instituciones considera el tipo de radionúclido como el criterio de segregación más importante, seguido por el estado físico con un $68 \%$, mientras que $73 \%$ segrega los desechos considerando el tipo de radiación emitida. La normatividad oficial mexicana y recomendaciones internacionales (NOM-028-NUCL, 2009; OIEA, 2000; OIEA, 1987) establecen que los desechos radiactivos deben colectarse y segregarse conforme al proceso de gestión al que será sometido; el criterio más generalizado es el estado físico: sólidos (compactables y no compactables) y líquidos (orgánicos y acuosos), entre otros. Estos pueden agruparse con otros desechos compatibles físicamente y que contengan radionúclidos de vida media similar, de tal forma que se optimice su gestión. Adicionalmente, en las aplicaciones médicas se utilizan materiales punzocortantes y biológicos, por lo que se generarán desechos mixtos, es decir, residuos peligrosos o biológico-infecciosos contaminados con radionúclidos. En estos casos se recomienda utilizar criterios de segregación adicionales. En términos generales, los resultados demostraron que las instituciones médicas realizan una segregación adecuada al considerar la naturaleza del radionúclido como el criterio de separación principal.

Respecto a las características físicas de los contenedores utilizados en la recolección de los desechos radiactivos, los resultados mostraron que $100 \%$ de las instituciones recolectan los materiales punzocortantes en contenedores rígidos que disminuyen los riesgos físicos del personal y facilitan el manejo posterior de los desechos en conformidad con la normatividad oficial mexicana (NOM-028-NUCL, 2009). Tal como se presenta en la gráfica 3, los contenedores utilizados para materiales como vendas, torundas o pañales cumplen de manera parcial con lo señalado en la NOM-028-NUCL (2009), la cual establece que los contenedores deben estar rotulados e identificados, así como tener un sistema para que pueda abrirse con un pie. Aunado a esto, los contenedores deben recubrirse con una bolsa plástica transparente, con franjas de color amarillo, resistente y biodegradable, que pueda ser sellada 
y retirada fácilmente cuando deje de utilizarse. Al respecto, 95\% de los hospitales identifican sus contenedores con el símbolo de radiación ionizante. Esta disposición es útil durante el proceso de segregación, ya que ayuda a conservar los materiales radiactivos separados de aquellos que no lo son. De los contenedores, $23 \%$ cuenta con un sistema de accionado con pedal con el fin de reducir el riesgo de su contaminación externa debido al contacto accidental con el material radiactivo, mientras que $77 \%$ restante no cuenta con estas adaptaciones ergonómicas. De igual manera, se considera que el contenedor sea de un tamaño adecuado que permita un fácil acceso a los desechos; esta característica se cumple en un 91\%. Asimismo, los contenedores deben cerrarse herméticamente para prevenir la dispersión de contaminación; esta disposición se cumple en un 50\%.

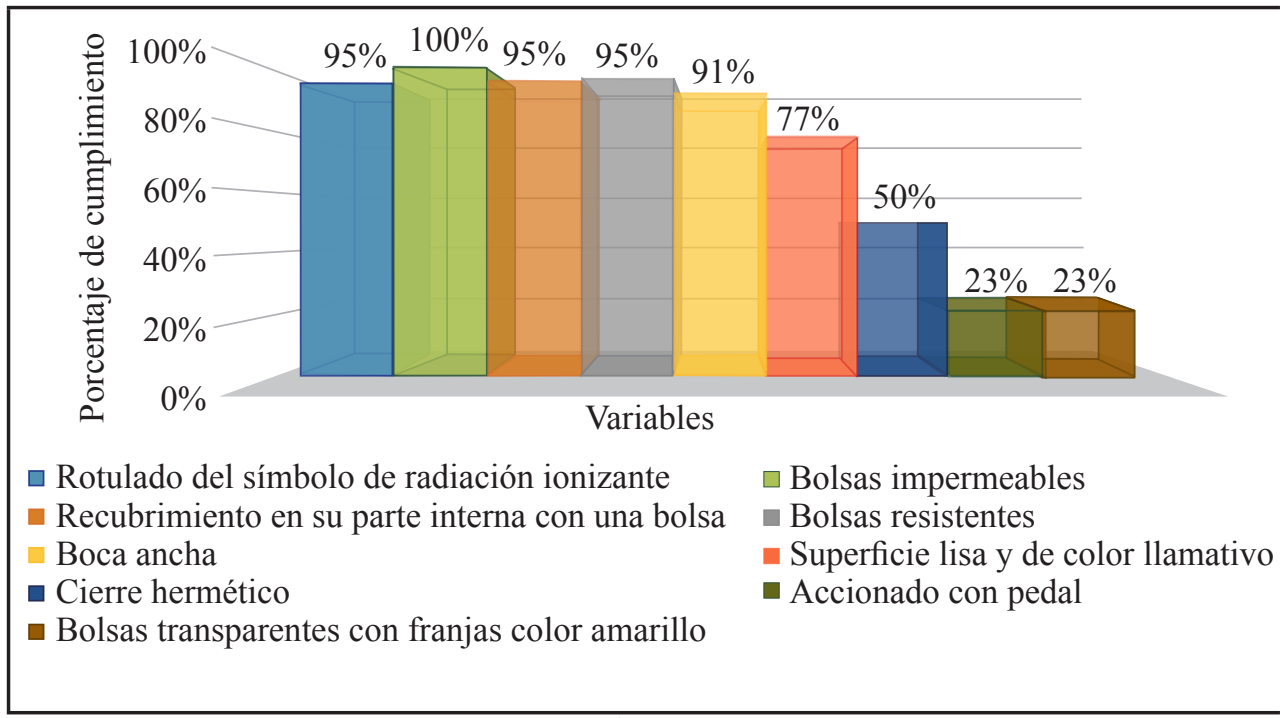

\section{GRÁFICA 3}

Características de los contenedores

Fuente: elaboración propia.

En referencia a los desechos líquidos radiactivos, se pudo determinar que $64 \%$ de las instituciones lo genera y sólo 14\% tiene contenedores apropiados:

a) El Instituto Nacional de Cancerología tiene un cárcamo donde realiza el monitoreo del drenaje caliente.

b) El Instituto Nacional de Ciencias Médicas y Nutrición Salvador Zubirán dispone de dos tanques de decaimiento blindados de aproximadamente $18 \mathrm{~m}^{3}$.

c) El Instituto Nacional de Enfermedades Respiratorias cuenta con un cárcamo para los desechos líquidos de unidosis.

En este aspecto, $50 \%$ de las instituciones no cuenta con este tipo de contenedores; algunos de los argumentos ante esta situación fueron que $a$ ) las excretas con desechos radiactivos se eliminan por el drenaje común junto con el agua residual que se genera en el hospital, $b$ ) existe una trampa en el drenaje donde se almacenan las excretas y se monitorean antes de descargarlas al drenaje general, $c$ ) se usa la técnica de dilución y $d$ ) va al drenaje y se mide mensualmente.

Las evidencias anteriores dejan ver la necesidad de mejorar los controles en la descarga de desechos líquidos y determinar la concentración de actividad o actividad radiactiva antes de realizar la dispensa. De acuerdo con el OIEA (2000,2013), este tipo de desechos puede descargarse tomando en cuenta dos principios dilución y dispersión y retención y decaimiento, los cuales proporciona dos opciones: a) la descarga directa al sistema de alcantarillado y $b$ ) el almacenamiento para su decaimiento previo a la descarga al sistema de alcantarillado. 
Para la descarga directa a un sistema de drenaje, los efluentes radiactivos deben de cumplir con los criterios de liberación (radiológicos, químicos y biológicos), además de que la infraestructura del alcantarillado debe de estar bien desarrollada, esto implica la existencia de plantas de tratamiento de aguas residuales. Cuando la infraestructura urbana no se encuentra bien desarrollada, se recomienda optar por el almacenamiento en contenedores o en la instalación de un sistema de tanques de decaimiento diseñados para reducir el impacto de los efluentes.

Otro elemento, muy ligado a la segregación y recolección, es el adecuado etiquetado y registro de los bultos que contienen los desechos radiactivos. En este sentido, la información recabada mostró que $95 \%$ de las instituciones señalan que etiquetan sus bultos. En referencia a la información que incluye la etiqueta, la NOM-028-NUCL (2009) y el artículo 85 del RGSR señalan que todo recipiente o contenedor debe incluir el rotulado de la información general del desecho. Esta información incluye fecha de retiro del área de generación, lugar donde se recolectó, radionúclido, actividad, concentración de actividad o actividad específica, nivel de radiación a contacto y a un metro, composición química o forma física, masa o volumen, símbolo de radiación ionizante y fecha final de la etapa de decaimiento. Los resultados obtenidos referentes al cumplimiento de los requerimientos del etiquetado mostraron que sólo cinco de nueve características tienen un porcentaje de cumplimiento razonable (77-95\%).

$\mathrm{Al}$ respecto, y en torno a la gestión de desechos radiactivos, las características que las instituciones médicas consideran como de mayor importancia para el etiquetado son fecha de generación, tipo de radionúclido, composición química/forma física, masa o volumen y fecha final de decaimiento. Asimismo, se tiene que 21 hospitales (95\%) registran la fecha en la que se genera el desecho; por otro lado, 91\% considera la fecha final de decaimiento. Estos dos aspectos son fundamentales en el proceso de gestión, ya que con base en ellos se determina el tiempo de almacenamiento temporal o decaimiento del desecho. Algo semejante sucede con el tipo de radionúclido, donde 21 hospitales (95\%) registran este aspecto. Esta información permite mantener homogeneidad de los desechos contaminados con cierto radionúclido. Referente a la composición química o forma física, 16 de los hospitales (73\%) coloca esta aclaración en la etiqueta. Por último, se tiene el aspecto de masa o volumen; esta información la rotulan únicamente 9 hospitales (41\%), lo cual deja ver que la mayoría no cuantifica los desechos radiactivos que producen y por consiguiente no tienen información confiable sobre su generación.

Respecto al registro de los desechos, la normatividad establece que los generadores deberán realizarlo en una bitácora que tiene como finalidad mantener el registro y control de la cantidad de desechos generados antes de que sean exentos de cualquier control regulador. Además, la norma oficial mexicana, que establece los requisitos de seguridad radiológica para la práctica de la medicina nuclear, define que las instalaciones que utilizan fuentes abiertas deben registrar el material administrado y desechado (NOM-040-NUCL, 2016). Esta información fue contrastada con lo obtenido en el cuestionario, de donde se puede determinar que $91 \%$ de las entidades consultadas mantienen el registro (bitácora de control) de los desechos radiactivos que ingresan al almacén temporal. En contraste, dos instituciones no realizan el registro de sus desechos, es decir, 9\%.

La mayoría de los hospitales cuenta con una bitácora de registro, por lo que se espera que contenga como mínimo la siguiente información: fecha de recepción y firma del responsable del almacén, fecha de generación del desecho, fecha de finalización de la etapa de decaimiento, radionúclido y su actividad, niveles de radiación a la fecha de recepción y la descripción del desecho radiactivo que especifique su composición química o forma física y su masa o volumen tal como lo establece la normatividad. El análisis de la información recabada mostró que 82\% de las instituciones registra la fecha de recepción del recipiente o bolsa, $86 \%$ registra en su bitácora el número de identificación del recipiente o bolsa de los desechos. El registro del tipo de radionúclido y su actividad se realiza en $82 \%$ de las instituciones. De igual manera, $82 \%$ de las instituciones anota el nivel de radiación a contacto y a un metro del recipiente o bolsa al momento de su recepción en el almacén. Finalmente, 59\% de las instituciones incluye una breve descripción de los desechos radiactivos contenidos en el recipiente o bolsa especificando su composición química o forma física, así como la masa o volumen. Cabe mencionar que no se obtuvo respuesta por parte de tres instituciones, ya que justifican que la información solicitada no aplica para su institución, pues consideran son acciones que se realizan en "almacenes definitivos". 
Los resultados dejan ver que, a pesar de que $91 \%$ de los hospitales tiene una bitácora de registro y control, la información que se consigna en ésta es incompleta. Cabe señalar que el objetivo más importante de la bitácora es la cuantificación y entradas o salidas de los desechos; sin embargo, nada más nueve instituciones inscriben esta información.

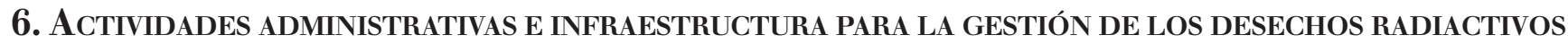

En general, la gestión de los desechos radiactivos comprende seis etapas: a) pretratamiento, $b$ ) tratamiento, $c$ ) acondicionamiento, $d$ ) almacenamiento, $e$ ) transporte y f) disposición final (OIEA, 2001). En el caso de los desechos radiactivos de origen hospitalario, con radionúclidos de vida media menor a un año, habitualmente se consideran tres etapas: $a$ ) pretratamiento, $b$ ) almacenamiento y $c$ ) dispensa (OIEA, 2001) como residuos no radiactivos (figura 2). Uno de los requerimientos más importantes para que los generadores realicen de manera correcta la gestión de los desechos es el desarrollo e implementación de un plan de gestión que permita minimizar la generación de los desechos, mantener una segregación eficiente y evitar la liberación de materiales contaminados con radionúclidos al ambiente. Bajo este contexto, de las 22 instituciones médicas, 50\% señaló que tiene un plan de gestión de desechos radiactivos, 50\% restante no cuenta con él, ya que los proveedores de los radiofármacos se hacen responsables de la gestión de los desechos generados. Es importante indicar que los proveedores sólo gestionan las jeringas provenientes de la aplicación de unidosis y no el material contaminado con dichos radionúclidos. Adicionalmente, los proveedores no están obligados de manera normativa a efectuar la gestión de los desechos por el uso de los radiofármacos; esta tarea supone una carga financiera para el proveedor que tarde o temprano pudiera no responsabilizarse de ellos.

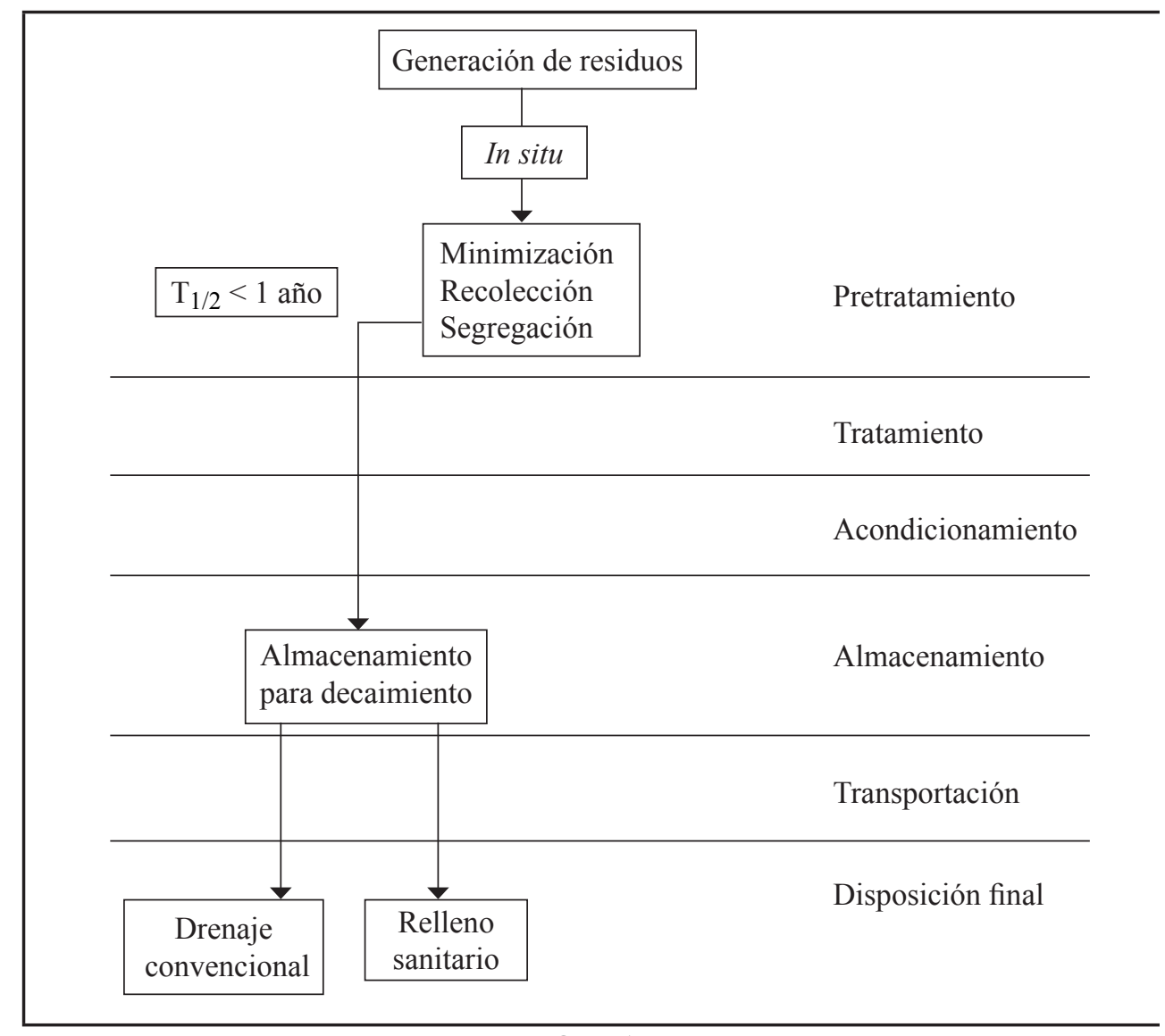

FIGURA 2

Etapas de gestión de los desechos radiactivos Fuente: elaboración propia con base en OIEA (2001). 
El plan de gestión con el que cuentan las instituciones médicas estudiadas consiste en un documento o procedimiento que indica que los desechos deberán dejarse decaer por 10 vidas medias o hasta que tengan niveles de radiación de fondo y puedan considerarse como residuos sólidos urbanos. Es importante remarcar que en el plan de gestión se establecen los procedimientos para que el manejo de los desechos sea acorde con la normatividad y permita prevenir y reducir riesgos al ambiente y salud pública. Con base en esta información, se puede determinar que las instituciones médicas no definen correctamente los procedimientos que conforman el plan de gestión, y por lo tanto pueden presentar dificultades en alguna de las tres etapas de gestión.

Respecto a la infraestructura que las instituciones médicas tienen para realizar la gestión de los desechos radiactivos, la información recabada mostró que 95\% tiene un almacén temporal, mientras que 5\% restante carece de uno. Ninguna institución realiza el tratamiento de desechos, sólo se efectúa el decaimiento radiactivo (10 vidas medias) en el almacén temporal. Finalizado el periodo de decaimiento, los desechos son manejados como residuos sólidos urbanos, aguas residuales, o bien como residuo peligroso o biológico infeccioso, según sea el caso.

Según la normatividad nacional y recomendaciones internacionales (NOM-028-NUCL, 2009; NOM-040NUCL, 2016; NOM-027-NUCL, 1996; OIEA, 2009), el área destinada para almacenamiento de los desechos radiactivos debe señalizarse con el símbolo internacional que advierte la presencia de radiación ionizante (95\% de las instituciones cumplen con este aspecto). Otra especificación señala que el área debe ser exclusivamente para este tipo de desechos, acción que se cumple en un 95\%. Asimismo, debe de estar aislado de las materias primas o materiales no radiactivos, disposición que se cumple en 91\%. Aunado a lo anterior, la capacidad de almacenaje debe de ser suficiente de acuerdo con lo generado por la institución, dicha disposición se cumple en un $95 \%$.

$\mathrm{Al}$ mismo tiempo, 68\% de las instituciones afirmaron que su almacén temporal se ubica en un lugar aislado del personal y de los pacientes y $95 \%$ refiere que su ubicación facilita la transferencia de los desechos del lugar de generación al sitio de decaimiento, de tal forma que se minimiza el transporte de material radiactivo dentro de la instalación. En cuanto a la restricción del acceso, 95\% de los hospitales cumple con esta disposición; de igual manera la estructura del almacén debe de ser hermética, sólo $73 \%$ de los hospitales lo considera. Otro requisito es que la puerta de acceso debe abrirse desde su interior sin necesidad de llave; $73 \%$ de los hospitales cumple con lo establecido. De igual manera, el almacén debe tener superficies lisas, impermeables y fácilmente descontaminables, situación que sólo $86 \%$ de las instituciones cumple. De acuerdo con los requerimientos establecidos, se tiene que los porcentajes obtenidos representan un cumplimiento aceptable en cuanto diseño y características de infraestructura de un almacén temporal.

Con base en los resultados obtenidos, se pudo evidenciar que la muestra de instituciones médicas estudiadas cumple parcialmente con la normatividad asociada a la gestión de sus desechos radiactivos; esto es motivado por la ausencia de un plan de gestión institucional que identifique los requerimientos normativos y de licenciamiento, que a su vez derive en la definición y descripción de las acciones operativas que garanticen su implementación y ejecución.

Este plan de gestión debe cumplir con las etapas señaladas en la figura 2: pretratamiento, almacenamiento y dispensa como residuos sólidos urbanos o peligrosos, según sea el caso. Este trabajo permitió identificar que los puntos que deben reforzarse en la etapa de pretratamiento son a) la planificación de los trabajos que involucren la manipulación de material radiactivo con la finalidad de adoptar medidas para la reducción de la cantidad de desechos generados, $b$ ) capacitar a los operadores para que separen, cuantifiquen e identifiquen adecuadamente los desechos dejando constancia de esta información en la etiqueta del bulto correspondiente y $c$ ) estimar el volumen y actividad de los desechos líquidos generados. En la etapa de almacenamiento se recomienda que las instituciones médicas realicen un registro (en bitácora) estricto de los bultos de desechos radiactivos que ingresan y salen del almacén temporal. Este registro debe incluir al menos la fecha de retiro del punto de generación, sitio de generación, radionúclido, actividad, composición, volumen o masa, símbolo y fecha estimada 
del final del decaimiento tal como lo establecen la normatividad y recomendaciones internacionales. Respecto a los desechos líquidos, dependiendo del volumen generado y de los escenarios de dilución considerados en el informe de seguridad de la instalación, la institución tendría que valorar la construcción de un sistema de decaimiento de líquidos. Finalmente, la etapa final de la gestión de los desechos es la dispensa incondicional. En este sentido, es importante que la institución, en función del análisis de información de la bitácora y del monitoreo de los desechos, identifique aquellos que han cumplido con el periodo de almacenamiento y han alcanzado los niveles de dispensa aprobados por el órgano regulador. Es recomendable que el plan de gestión considere las acciones para garantizar que los desechos dispensados sean depuestos conforme a las regulaciones ambientales aplicables, ya sea como residuos sólidos urbanos, residuos de manejo especial o residuos peligrosos (biológico-infecciosos), según sea el caso.

\section{Conclusiones}

La evidencia obtenida permitió identificar que las instituciones médicas estudiadas cumplen de manera parcial con la normatividad asociada a la gestión de sus desechos radiactivos sin que estas áreas de oportunidad impliquen un riesgo radiológico para el personal ocupacionalmente expuesto, pacientes o el ambiente. La indefinición de un plan de gestión institucional es el agente causal de las áreas de oportunidad observadas.

Medidas de capacitación, cuantificación e identificación de los desechos son requeridas en la etapa del pretramiento. En la etapa de almacenamiento es necesario incidir en el registro (en bitácora) de los bultos que ingresan y salen del almacén temporal. Se identificó la necesidad de establecer los procedimientos de dispensa adecuados de los desechos decaídos, así como garantizar que el plan de gestión considere las acciones necesarias para que los desechos dispensados sean evacuados conforme a las regulaciones ambientales aplicables. Respecto a los desechos líquidos, es necesario su cuantificación y estimación de actividad para definir si es requerida la construcción de sistemas de decaimiento de líquidos.

\section{Análisis Prospectivo}

México es un país donde más de $80 \%$ de las instituciones médicas públicas con unidades de medicina nuclear

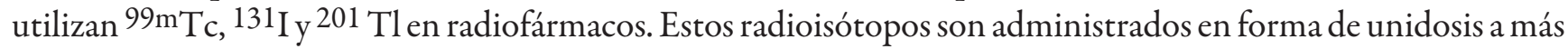
de 200 pacientes al mes, lo que conlleva a que el sector salud sea el principal generador de desechos radiactivos en el país. En este contexto, este trabajo pone de manifiesto que las instituciones médicas estudiadas cumplen de modo parcial con la normatividad asociada a la gestión de sus desechos radiactivos sin que estas áreas de oportunidad impliquen un riesgo radiológico para el personal ocupacionalmente expuesto, pacientes o el ambiente. Sin embargo, el panorama actual de la demanda de los radioisótopos en el diagnóstico y tratamiento de enfermedades de diferente índole evidencian que la generación de desechos radiactivos de vida media corta se incrementará sustancialmente en los próximos años y con ello los riesgos de ocurrencia de un evento no deseado si se persiste en mantener las deficiencias identificadas. Por lo tanto, es indispensable que las instituciones médicas y el sector salud en general mejoren los procedimientos operacionales necesarios en cada una de las etapas de gestión de los desechos radiactivos con la finalidad de mantener al mínimo el riesgo radiológico, ambiental y de salud ocupacional. Las acciones como el desarrollo e implementación de un plan de gestión, capacitación y supervisión son necesarias en el corto plazo.

\section{Agradecimientos}

Los autores agradecen a la Secretaría de Salud y a la Comisión Nacional de Seguridad Nuclear y Salvaguardias por la información proporcionada. 


\section{ReFERENCIAS}

Álvarez, A. y Moreno, L. (2010). Plan de gestión integral de residuos hospitalarios y similares componente interno para una institución prestadora de servicios de salud de primer nivel (tesis de licenciatura). Colombia: Universidad Industrial de Santander.

Álvarez, Rico, Y. (2010). Caracterización de desechos contaminados con material radiactivo en medicina nuclear (tesis). México: Instituto Politécnico Nacional.

Comisión Nacional de Seguridad Nuclear y Salvaguardias (CNSNS). (2015). Solicitud de información a través de la plataforma nacional de transparencia del Instituto Nacional de Transparencia, Acceso a la Información y Protección de Datos Personales (INAI).

Cortés, A. y Esteban, J. (2003). Radiofármacos de uso humano: marco legal e indicaciones clínicas autorizadas en España. Seguridad Nuclear, 26, 5-15. Disponible en https://www.aemps.gob.es/publicaciones/articulo/docs/radiofarmacos_uso_humano.pdf

Chain, Y. e Illanes, L. (2015). Radiofármacos en medicina nuclear fundamentos y aplicación clinica. La Plata: Universidad Nacional de la Plata. Disponible en http://sedici.unlp.edu.ar/bitstream/ handle/10915/46740/Documento_completo.pdf?sequence $=1$

Díaz, A. (2015). Renovarse para seguir trabajando. Apps Nucleares, 1, 19. Disponible en http://www.inin. gob.mx/documentos/publicaciones/Apps_Nucleares/Apps\%20Nucleares_1.pdf

Duran, E. y Munar, P. (2006). Diagnóstico de seguridad ambiental para la gestión de residuos radiactivos generados por la medicina nuclear en Bogotá D.C. (tesis). Bogotá: Universidad de La Salle.

Escofet, A., Folgueiras, P. Luna, E. y Palou, B. (2016). Elaboración y validación de un cuestionario para la valoración de proyectos de aprendizaje-servicio. Revista Mexicana de Investigación Educativa, 21(70), 929-949.

Galtung, J. (1967). Theory and methods of social research. New York: Columbia University Press.

Henriques, S. (2014). Seven things to know about radioisotopes. OIEA Boletin, 55(4), 8-9. Disponible en https://www.iaea.org/sites/default/files/55405810809.pdf

Jawerth, N. (2014). Radiation and radionuclides in medicine a brief overview of nuclear medicine and radiotherapy. OIEA Boletín, 55(4), 5-7. Disponible en https://www.iaea.org/sites/default/files/55405810507.pdf

Machi, S., Sevastyanoy, Y. G., \&Yuan H. C. (1983). Isotopes and radiation for modern industry. OIEA Boletín, 25(1), 11-14. Disponible en https://www.iaea.org/sites/default/files/publications/magazines/bulletin/ bull25-1/25105391114.pdf.

Ministerio de Sanidad Servicios Sociales e Igualdad (MSSSI) (1996). Medicina Nuclear. Disponible en http:// www.msssi.gob.es/profesionales/formacion/docs/Medicina_Nuclear.pdf

NOM-027-NUCL-1996. (1997). Especificaciones para el diseño de instalaciones radiactivas Tipo II Clases A, B y $C$. Diario Oficial de la Federación.

NOM-028-NUCL-2009. (2009). Manejo de desechos radiactivos en instalaciones radiactivas que utilizan fuentes abiertas. Diario Oficial de la Federación.

NOM-040-NUCL-2016. (2016). Requisitos de seguridad radiológica para la práctica de medicina nuclear. Diario Oficial de la Federación.

OIEA (Organismo Internacional de Energía Atómica).(2009). Gestión de desechos procedentes de la utilización de materiales radiactivos en medicina, industria, agricultura, investigación y educación (Guía de seguridad No. WS-G-2.7). Viena. 
OIEA (Organismo Internacional de Energía Atómica). (2001). Handling and processing of radiactive waste from nuclear applications (Technical Report No. 402). Viena.

OIEA (Organismo Internacional de Energía Atómica). (2015). Los átomos en la industria tecnología de la radiación para el desarrollo. OIEA Boletin, 56(3), 4-5. Disponible en https://www.iaea.org/sites/ default/files/bull563_sept2015_es.pdf

OIEA (Organismo Internacional de Energía Atómica). (2013). Management of Discharge of low level liquid radioactive waste generated in medical, educational, research and industrial facilities (TECDOC-1714). Viena.

OIEA (Organismo Internacional de Energía Atómica). (2000). Management of radioactive waste from the use of radionuclides in medicine (TECDOC-1183). Viena.

OIEA (Organismo Internacional de Energía Atómica). (1987). Techniques and Practices for Pretreatment of Low and Intermediate Level Solid and Liquid Radioactive Waste (Technical Report No. 272). Viena.

Ortiz, H. B. (2002). Tratamiento de aguas oleosas por flotación (tesis). Toluca: Universidad Autónoma del Estado de México.

Pichardo, P. (2015). Normatividad y vigilancia radiológica. Familia Médica, 35, 21-23. Disponible en http:// www.imagenglobal.org/wp-content/uploads/2015/11/familia_medica_37.pdf.

Skromne, K. G. (2010) Medicina nuclear. Desarrollo histórico e impacto en la salud. Revista Hospital Juárez de México, 77(4), 295-297. Disponible en http://www.medigraphic.com/pdfs/juarez/ju-2010/ju104w.pdf.

Villareal, J. (2011) Energía nuclear en México. Heinrich Böll-México Centroamérica yel Caribe, 1-4. Disponible en https://mx.boell.org/sites/default/files/downloads/Articulo_nuclear_en_Mexico.pdf.

\section{BY-NC-ND}

